Valeriu Popa, Department of Mathematics, University of Bacău, 5500 Bacău, Romania

Takashi Noiri, Mathematics Department, Yatsushiro College of Technology, Yatsushiro, Kumamoto, 866 Japan

\title{
ON UPPER AND LOWER $\beta$-CONTINUOUS MULTIFUNCTIONS
}

\begin{abstract}
In this paper the authors define a multifunction $F: X \mapsto Y$ to be upper (respectively, lower) $\beta$-continuous if $F^{+}(V)$ (resp. $F^{-}(V)$ ) is $\beta$ open in $X$ for every open set $V$ of $Y$. They obtain some characterizations and several properties concerning upper (resp. lower) $\beta$-continuous multifunctions. The relationships between these multifunctions and quasi continuous multifunctions are investigated.
\end{abstract}

\section{Introduction}

Abd El-Monsef et al. [1] defined $\beta$-continuous functions as a generalization of semi-continuity [15] and precontinuity [17]. Recently, Borsík and Doboš [7] have introduced the notion of almost quasi- continuity which is weaker than that of quasi-continuity [16] and obtained a decomposition theorem of quasicontinuity. The authors [26] of the present paper obtained several characterizations of $\beta$-continuity and showed that almost quasi-continuity is equivalent to $\beta$-continuity. The equivalence of almost quasi-continuity and $\beta$-continuity is also shown by Borsík [6] and Ewert [11]. The purpose of the present paper is to define upper (lower) $\beta$-continuous multifunctions and to obtain several characterizations of upper (lower) $\beta$-continuous multifunctions and several properties of such multifunctions.

Key Words: $\beta$-open, $\beta$-continuous, almost quasicontinuous, quasicontinuous, multifunctions, nets for multifunctions

Mathematical Reviews subject classification: 54C10; 54C60

Received by the editors September 15, 1995

* The authors would like to thank the referee for providing valuable comments and suggestions. 


\section{Preliminaries}

Let $X$ be a topological space and $A$ a subset of $X$. The closure of $A$ and the interior of $A$ are denoted by $\mathrm{Cl}(A)$ and $\operatorname{Int}(A)$, respectively. A subset $A$ is said to be $\alpha$-open [19] (resp. semi-open [15], preopen [17], $\beta$-open [1] or semipreopen [3]) if $A \subset \operatorname{Int}(\mathrm{Cl}(\operatorname{Int} A)))$ (resp. $A \subset \mathrm{Cl}(\operatorname{Int}(A)), A \subset \operatorname{Int}(\mathrm{Cl}(A))$, $A \subset \mathrm{Cl}(\operatorname{Int}(\mathrm{Cl}(A))))$. The family of all semi-open (resp. $\beta$-open) sets of $X$ containing a point $x \in X$ is denoted by $S O(X, x)$ (resp. $\beta(X, x)$ ). The family of all $\alpha$-open (resp. semi-open, preopen, semi-preopen) sets in $X$ is denoted by $\alpha(X)$ (resp. $S O(X), P O(X), \beta(X)$ ). For these four families, it is shown in [20] (see Lemma 3.1) that $S O(X) \cap P O(X)=\alpha(X)$, and it is obvious that $S O(X) \cup P O(X) \subset \beta(X)$. The complement of an $\alpha$-open (resp. semi-open, preopen, $\beta$-open) set is said to be $\alpha$-closed (resp. semi-closed [9], preclosed [10], $\beta$-closed [1]). The intersection of all $\beta$-closed sets of $X$ containing $A$ is called the $\beta$-closure [2] of $A$, and it is denoted by $\beta \operatorname{Cl}(A)$. Similarly, $\alpha \mathrm{Cl}(A)$, $s \mathrm{Cl}(A)$ and $p \operatorname{Cl}(A)$ are defined. The union of all $\beta$-open sets of $X$ contained in $A$ is called the $\beta$-interior of $A$ and is denoted by $\beta \operatorname{Int}(A)$. Abd El-Monsef et al. [1] defined a function to be $\beta$-continuous if the inverse image of every open set is $\beta$-open.

Throughout this paper, spaces $(X, \tau)$ and $(Y, \sigma)$ (or simply $X$ and $Y$ ) always mean topological spaces, and $F: X \mapsto Y$ (resp. $f: X \mapsto Y$ ) represents a multivalued (resp. single valued) function. For a multifunction $F: X \mapsto Y$, we shall denote the upper and lower inverse of a set $G$ of $Y$ by $F^{+}(G)$ and $F^{-}(G)$, respectively, that is $F^{+}(G)=\{x \in X: F(x) \subset G\}$ and $F^{-}(G)=$ $\{x \in X: F(x) \cap G \neq \emptyset\}$.

\section{Characterizations}

Definition 3.1 A multifunction $F: X \mapsto Y$ is said to be

(a) upper $\beta$-continuous [27] (briefly u. $\beta$.c.) at a point $x \in X$ if for each open set $V$ containing $F(x)$, there exists $U \in \beta(X, x)$ such that $F(U) \subset V$;

(b) lower $\beta$-continuous [27] (briefly l. $\beta . c$.$) at a point x \in X$ if for each open set $V$ such that $F(x) \cap V \neq \emptyset$, there exists $U \in \beta(X, x)$ such that $U \subset F^{-}(V)$;

(c) upper (lower) $\beta$-continuous if $F$ has this property at every point of $X$.

Remark 3.1 According to the referee, the definition of the upper and lower $\beta$-continuity at a point can be found in [12], where for the definition it is taken the condition (e) from Theorem 3.1 (resp. Theorem 3.2).

Theorem 3.1 The following are equivalent for a multifunction $F: X \mapsto Y$ : 
(a) $F$ is u.ß.c. at a point $x \in X$;

(b) for each open neighborhood $U$ of $x$ and each open set $V$ of $Y$ with $x \in$ $F^{+}(V), F^{+}(V) \cap U$ is not nowhere dense;

(c) for each open neighborhood $U$ of $x$ and each open set $V$ of $Y$ with $x \in$ $F^{+}(V)$, there exists an open set $G$ of $X$ such that $\emptyset \neq G \subset U$ and $G \subset$ $C l\left(F^{+}(V)\right)$;

(d) for each open set $V$ of $Y$ with $x \in F^{+}(V)$, there exists $U \in S O(X, x)$ such that $U \subset C l\left(F^{+}(V)\right)$;

(e) $x \in C l\left(\operatorname{Int}\left(C l\left(F^{+}(V)\right)\right)\right)$ for every open set $V$ of $Y$ with $x \in F^{+}(V)$.

Proof. (a) $\Rightarrow$ (b) and (b) $\Rightarrow$ (c): The proofs are obvious and are thus omitted. (c) $\Rightarrow(\mathrm{d})$ : Let $V$ be an open set of $Y$ containing $F(x)$. By $\mathcal{U}(x)$ we denote the family of all open neighborhoods of $x$. For each $U \in \mathcal{U}(x)$, there exists an open set $G_{U}$ of $X$ such that $\emptyset \neq G_{U} \subset U$ and $G_{U} \subset \mathrm{Cl}\left(F^{+}(V)\right)$. Put $W=\cup\left\{G_{U}: U \in \mathcal{U}(x)\right\}$. Then $W$ is an open set of $X, x \in \mathrm{Cl}(W)$ and $W \subset \mathrm{Cl}\left(F^{+}(V)\right)$. Moreover, we put $U_{o}=W \cup\{x\}$. Then $W \subset U_{o} \subset \mathrm{Cl}(W)$ and $U_{o} \in S O(X, x)$ and also $U_{o} \subset \mathrm{Cl}\left(F^{+}(V)\right)$.

(d) $\Rightarrow$ (e): Let $V$ be an open set of $Y$ containing $F(x)$. There exists $U \in S O(X, x)$ such that $U \subset \mathrm{Cl}\left(F^{+}(V)\right)$. Therefore, we have $x \in U \subset$ $\mathrm{Cl}(\operatorname{Int}(U)) \subset \mathrm{Cl}\left(\operatorname{Int}\left(\mathrm{Cl}\left(F^{+}(V)\right)\right)\right)$.

(e) $\Rightarrow$ (a): This is shown in [12].

Theorem 3.2 The following are equivalent for a multifunction $F: X \mapsto Y$ :

(a) $F$ is l.ß.c. at a point $x \in X$;

(b) for any open neighborhood $U$ of $x$ and any open set $V$ of $Y$ with $x \in$ $F^{-}(V), F^{-}(V) \cap U$ is not nowhere dense;

(c) for any open neighborhood $U$ of $x$ and any open set $V$ of $Y$ with $x \in$ $F^{-}(V)$, there exists an open set $G$ of $X$ such that $\emptyset \neq G \subset U$ and $G \subset$ $C l\left(F^{-}(V)\right)$;

(d) for any open set $V$ of $Y$ with $x \in F^{-}(V)$, there exists $U \in S O(X, x)$ such that $U \subset C l\left(F^{-}(V)\right)$;

(e) $x \in C l\left(\operatorname{Int}\left(C l\left(F^{-}(V)\right)\right)\right)$ for every open set $V$ of $Y$ with $x \in F^{-}(V)$.

Proof. The proof is similar to that of Theorem 3.1.

Theorem 3.3 The following are equivalent for a multifunction $F: X \mapsto Y$ : 
(a) F is u.ß.c.,;

(b) $F^{+}(V) \in \beta(X)$ for every open set $V$ of $Y$;

(c) $F^{-}(K)$ is $\beta$-closed in $X$ for every closed set $K$ of $Y$;

(d) ${ }_{\beta} C l\left(F^{-}(B)\right) \subset F^{-}(C l(B))$ for every subset $B$ of $Y$;

(e) $\operatorname{Int}\left(C l\left(\operatorname{Int}\left(F^{-}(B)\right)\right)\right) \subset F^{-}(C l(B))$ for every subset $B$ of $Y$.

Proof. $(\mathrm{a}) \Rightarrow(\mathrm{b})$ : Let $V$ be any open set of $Y$ and $x \in F^{+}(V)$. There exists $U \in \beta(X, x)$ such that $F(U) \subset V$. Therefore, we obtain $x \in U \subset$ $\mathrm{Cl}(\operatorname{Int}(\mathrm{Cl}(U))) \subset \mathrm{Cl}\left(\operatorname{Int}\left(\mathrm{Cl}\left(F^{+}(V)\right)\right)\right)$. Then $F^{+}(V) \subset \mathrm{Cl}\left(\operatorname{Int}\left(\mathrm{Cl}\left(F^{+}(V)\right)\right)\right)$ and hence $F^{+}(V) \in \beta(X)$.

$(\mathrm{b}) \Rightarrow(\mathrm{c})$ : This follows immediately from the fact that $F^{+}(Y \backslash B)=$ $X \backslash F^{-}(B)$ for every subset $B$ of $Y$.

$(\mathrm{c}) \Rightarrow(\mathrm{d})$ : For any subset $B$ of $Y, \mathrm{Cl}(B)$ is closed in $Y$ and $F^{-}(\mathrm{Cl}(B))$ is $\beta$-closed in $X$. Therefore, we obtain $\beta \mathrm{Cl}\left(F^{-}(B)\right) \subset F^{-}(B)$.

$(\mathrm{d}) \Rightarrow(\mathrm{e})$ : Let $B$ be any subset of $Y$. By [3] (see Theorem 2.15), we obtain $\operatorname{Int}\left(\mathrm{Cl}\left(\operatorname{Int}\left(F^{-}(B)\right)\right)\right) \subset \beta \mathrm{Cl}\left(F^{-}(B)\right) \subset F^{-}(\mathrm{Cl}(B))$.

$(\mathrm{e}) \Rightarrow(\mathrm{b})$ : Let $V$ be any open set of $Y$. Then $Y \backslash V$ is closed in $Y$ and we have $X \backslash F^{+}(V)=F^{-}(Y \backslash V) \supset \operatorname{Int}\left(\mathrm{Cl}\left(\operatorname{Int}\left(F^{-}(Y \backslash V)\right)\right)\right)=\operatorname{Int}(\operatorname{Cl}(\operatorname{Int}(X \backslash$ $\left.\left.\left.\left.F^{+}(V)\right)\right)\right)=X \backslash \mathrm{Cl}\left(\operatorname{Int}\left(F^{+}(V)\right)\right)\right)$. We obtain $F^{+}(V) \subset \mathrm{Cl}\left(\operatorname{Int}\left(\mathrm{Cl}\left(F^{+}(V)\right)\right)\right)$ and hence $F^{+}(V) \in \beta(V)$.

(b) $\Rightarrow(\mathrm{a})$ : Let $x \in X$ and $V$ be an open set of $Y$ containing $F(x)$. By (b), we have $x \in F^{+}(V) \in \beta(X)$. Put $U=F^{+}(V)$. Then we obtain $U \in \beta(X, x)$ and $F(U) \subset V$. Therefore, $F$ is upper $\beta$-continuous.

Theorem 3.4 The following are equivalent for a multifunction $F: X \mapsto Y$ :

(a) F is l.ß.c.,;

(b) $F^{-}(V) \in \beta(X)$ for every open set $V$ of $Y$;

(c) $F^{+}(K)$ is $\beta$-closed in $X$ for every closed set $K$ of $Y$;

(d) $\beta C l\left(F^{+}(B)\right) \subset F^{+}(C l(B))$ for every subset $B$ of $Y$;

(e) $\operatorname{Int}\left(C l\left(\operatorname{Int}\left(F^{+}(B)\right)\right)\right) \subset F^{+}(C l(B))$ for every subset $B$ of $Y$.

(f) $F(\operatorname{Int}(C l(\operatorname{Int}(A)))) \subset C l(F(A))$ for every subset $A$ of $X$;

(g) $F(\beta C l(A)) \subset C l(F(A))$ for every subset $A$ of $X$. 
Proof. It is shown similarly to the proof of Theorem 3.3 that the statements (a), (b), (c), (d) and (e) are equivalent. We shall prove only the following implications.

(e) $\Rightarrow(\mathrm{f})$ : Let $A$ be any subset of $X$. Then we have $\operatorname{Int}(\mathrm{Cl}(\operatorname{Int}(A))) \subset$ $\operatorname{Int}\left(\mathrm{Cl}\left(\operatorname{Int}\left(F^{+}(F(A))\right)\right)\right) \subset F^{+}(\mathrm{Cl}(F(A)))$. Therefore $F(\operatorname{Int}(\mathrm{Cl}(\operatorname{Int}(A)))) \subset$ $\mathrm{Cl}(F(A))$.

(f) $\Rightarrow$ (g): Let $A$ be any subset of $X$. By [3] (see Theorem 2.15), we have $F(\beta \mathrm{Cl}(A))=F(A \cup \operatorname{Int}(\mathrm{Cl}(\operatorname{Int}(A))))=F(A) \cup F(\operatorname{Int}(\mathrm{Cl}(\operatorname{Int}(A)))) \subset \mathrm{Cl}(F(A))$.

$(\mathrm{g}) \Rightarrow(\mathrm{c})$ : Let $K$ be any closed set of $Y$. Then we have $F\left({ }_{\beta} \mathrm{Cl}\left(F^{+}(K)\right)\right) \subset$ $\mathrm{Cl}\left(F\left(F^{+}(K)\right)\right) \subset \mathrm{Cl}(K)=K$. Therefore, we have $\beta \mathrm{Cl}\left(F^{+}(K)\right) \subset F^{+}(K)$, and hence $F^{+}(K)$ is $\beta$-closed in $X$.

For a multifunction $F: X \mapsto Y$, the graph multifunction $G_{F}: X \mapsto X \times Y$ is defined as follows: $G_{F}(x)=\{x\} \times F(x)$ for every $x \in X$.

Lemma 3.1 (Noiri and Popa, [22]) For a multifunction $F: X \mapsto Y$, the following hold:

$$
\text { (a) } G_{F}^{+}(A \times B)=A \cap F^{+}(B) \text { and (b) } G_{F}^{-}(A \times B)=A \cap F^{-}(B)
$$

for any subsets $A \subset X$ and $B \subset Y$.

Theorem 3.5 Let $F: X \mapsto Y$ be a multifunction such that $F(x)$ is compact

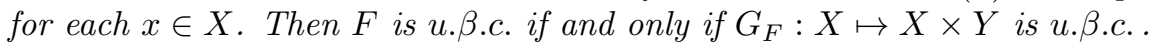

Proof. Necessity. Suppose that $F: X \mapsto Y$ is u.ß.c. . Let $x \in X$ and $W$ be any open set of $X \times Y$ containing $G_{F}(X)$. For each $y \in F(x)$, there exist open sets $U(y) \subset X$ and $V(y) \subset Y$ such that $(x, y) \in U(y) \times V(y) \subset W$. The family $\{V(y): y \in F(x)\}$ is an open cover of $F(x)$, and $F(x)$ is compact. Therefore, there exist a finite number of points $y_{1}, y_{2}, \ldots, y_{n}$ in $F(x)$ such that $F(x) \subset \cup\left\{V\left(y_{i}\right): 1 \leq i \leq n\right\}$. Set $U=\cap\left\{U\left(y_{i}\right): 1 \leq i \leq n\right\}$ and $V=\cup\left\{V\left(y_{i}\right): 1 \leq i \leq n\right\}$. Then $U$ and $V$ are open in $X$ and $Y$, respectively, and $\{x\} \times F(x) \subset U \times V \subset W$. Since $F$ is u. $\beta$.c., there exists $U_{o} \in \beta(X, x)$ such that $F\left(U_{o}\right) \subset U$. By Lemma 3.1, we have $U \cap U_{o} \subset U \cap F^{+}(V)=G_{F}^{+}(U \times V) \subset$ $G_{F}^{+}(W)$. Therefore, we obtain $U \cap U_{o}=\beta(X, x)$ and $G_{F}\left(U \cap U_{o}\right) \subset W$. This shows that $G_{F}$ is u. $\beta . c$.

Strong sufficiency. Suppose that $G_{F}: X \mapsto X \times Y$ is u. $\beta . c$. . Let $x \in X$ and $V$ be any open set of $Y$ containing $F(x)$. Since $X \times V$ is open in $X \times Y$ and $G_{F}(x) \subset X \times V$, there exists $U \in \beta(X, x)$ such that $G_{F}(U) \subset X \times V$. By Lemma 3.1, we have $U \subset G_{F}^{+}(X \times V)=F^{+}(V)$ and $F(U) \subset V$. This shows that $F$ is u. . .c. .

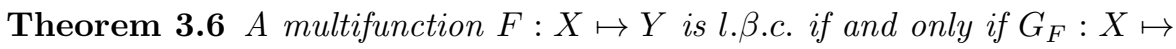
$X \times Y$ is l. $\beta . c$. . 
Proof. Necessity. Suppose that $F$ is l.ß.c. . Let $x \in X$ and $W$ be any open set of $X \times Y$ such that $x \in G_{F}^{-}(W)$. Since $W \cap(\{x\} \times F(x)) \neq \emptyset$, there exists $y \in F(x)$ such that $(x, y) \in W$, and hence $(x, y) \in U \times V \subset W$ for some open sets $U \subset X$ and $V \subset Y$. Since $F(x) \cap V \neq \emptyset$, there exists $G \in \beta(X, x)$ such that $G \subset F^{-}(V)$. By Lemma 3.1, we have $U \cap G \subset U \cap F^{-}(V)=G_{F}^{-}(U \times V) \subset$

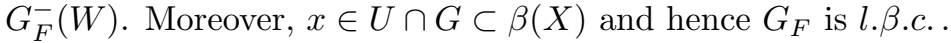

Sufficiency. Suppose that $G_{F}$ is l. $\beta$.c. . Let $x \in X$ and $V$ be an open set of $Y$ such that $x \in F^{-}(V)$. Then $X \times V$ is open in $X \times Y$ and $G_{F}(x) \cap(X \times V)=$ $(\{x\} \times F(x)) \cap(X \times V)=\{x\} \times(F(x) \cap V) \neq \emptyset$. Since $G_{F}$ is l. $\beta . c$. , there exists $U \in \beta(X, x)$ such that $U \subset G_{F}^{-}(X \times V)$. By Lemma 3.1, we obtain $U \subset F^{-}(V)$. This shows that $F$ is l. $\beta . c$. .

Definition 3.2 A subset $A$ of a topological space $X$ is said to be

(a) $\alpha$-paracompact [28] if every cover of $A$ by open sets of $X$ is refined by a cover of $A$ which consists of open sets of $X$ and is locally finite in $X$;

(b) $\alpha$-regular [14] if for each $a \in A$ and each open set $U$ of $X$ containing a there exists an open set $G$ of $x$ such that $a \in G \subset C l(G) \subset U$.

Lemma 3.2 (Kovacević [14]) If $A$ is an $\alpha$-regular $\alpha$-paracompact set of a topological space $X$ and $U$ is an open neighborhood of $A$, then there exists an open set $G$ of $X$ such that $A \subset G \subset C l(G) \subset U$.

For a multifunction $F: X \mapsto Y$, by ClF : $X \mapsto Y$ (see [4]) we denote a multifunction defined as follows: $(\mathrm{ClF})(x)=\mathrm{Cl}(F(x))$ for each $x \in X$. Similarly, we can define $\beta \mathrm{ClF}: X \mapsto Y, s \mathrm{ClF}: X \mapsto Y, p \mathrm{ClF}: X \mapsto Y$ and ${ }_{\alpha} \mathrm{ClF}: X \mapsto Y$.

Lemma 3.3 If $F: X \mapsto Y$ is a multifunction such that $F(x)$ is $\alpha$-paracompact $\alpha$-regular for each $x \in X$, then for each open set $V$ of $Y, G^{+}(V)=F^{+}(V)$, where $G$ denotes $\beta C l F, s C l F, p C l F, \alpha C l F$ or $C l F$.

Proof. Let $V$ be any open set of $Y$. Let $x \in G^{+}(V)$. Then $G(x) \subset V$ and $F(x) \subset G(x) \subset V$. We have $x \in F^{+}(V)$, and hence $G^{+}(V) \subset F^{-}(V)$. Conversely, let $x \in F^{+}(V)$, then $F(x) \subset V$. By Lemma 3.2, there exists an open set $H$ of $Y$ such that $F(x) \subset H \subset \mathrm{Cl}(H) \subset V$; hence $G(x) \subset \mathrm{Cl}(H) \subset V$. Therefore, we have $x \in G^{+}(V)$ and $F^{+}(V) \subset G^{+}(V)$.

Theorem 3.7 Let $F: X \mapsto Y$ be a multifunction such that $F(x)$ is $\alpha$ paracompact and $\alpha$-regular for each $x \in X$. Then the following are equivalent:

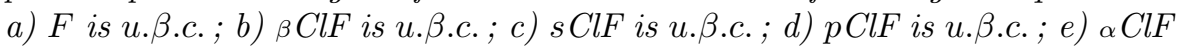

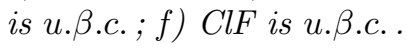


Proof. Similarly to Lemma 3.3, we put $G=\beta \mathrm{ClF}, s \mathrm{ClF}, p \mathrm{ClF}, \alpha \mathrm{ClF}$ or ClF.

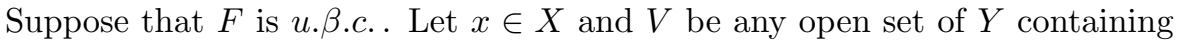
$G(x)$. By Lemma 3.3, $x \in G^{+}(V)=F^{+}(V)$ and there exists $U \in \beta(X, x)$ such that $F(U) \subset V$. Since $F(u)$ is $\alpha$-paracompact and $\alpha$-regular for each $u \in U$, by Lemma 3.2 there exists an open set $H$ such that $F(u) \subset H \subset \mathrm{Cl}(H) \subset V$; hence $G(u) \subset \mathrm{Cl}(H) \subset V$ for each $u \in U$. Therefore, we obtain $G(U) \subset V$. This shows that $G$ is u. $\beta . c$. .

Conversely, suppose that $G$ is u. . .c. . Let $x \in X$ and $V$ be any open set of $Y$ containing $F(x)$. By Lemma 3.3, $x \in F^{+}(V)=G^{+}(V)$ and hence $G(x) \subset V$. There exists $U \in \beta(X, x)$ such that $G(U) \subset V$. Thus $U \subset G^{+}(V)=F^{+}(V)$,

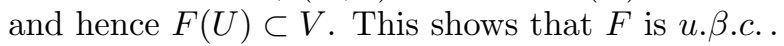

Lemma 3.4 If $F: X \mapsto Y$ is a multifunction, then for each open set $V$ of $Y$, $G^{-}(V)=F^{-}(V)$, where $G$ denotes $\beta C l F, s C l F, p C l F, \alpha C l F$ or $C l F$.

Proof. Let $V$ be any open set of $Y$ and $x \in G^{-}(V)$. Then $G(x) \cap V \neq \emptyset$, and hence $F(x) \cap V \neq \emptyset$ since $V$ is open. Thus, we obtain $x \in F^{-}(V)$ and hence $G^{-}(V) \subset F^{-}(V)$. Conversely, let $x \in F^{-}(V)$. Then we have $\emptyset \neq$ $F(x) \cap V \subset G(x) \cap V$ and hence $x \in G^{-}(V)$. Thus, we have $F^{-}(V) \subset G^{-}(V)$. Consequently, we obtain $G^{-}(V)=F^{-}(V)$.

Theorem 3.8 For a multifunction $F: X \mapsto Y$, the following are equivalent:

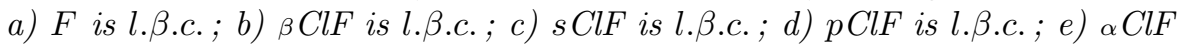

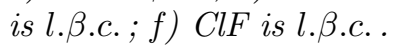

Proof. By using Lemma 3.4, this is shown similarly as in Theorem 3.7.

Theorem 3.9 Let $\left\{U_{\alpha}: \alpha \in \nabla\right\}$ be an $\alpha$-open cover of a topological space $X$. A multifunction $F: X \mapsto Y$ is u. $\beta$.c. if and only if the restriction $F / U_{\alpha}$ : $U_{\alpha} \mapsto Y$ is u. $\beta$.c. for each $\alpha \in \nabla$.

Proof. Necessity. Let $\alpha \in \nabla$ and $x \in U_{\alpha}$. Let $V$ be an open set of $Y$ such that $\left(F / U_{\alpha}\right)(x) \subset V$. Since $F$ is u. . .c. and $F(x)=\left(F / U_{\alpha}\right)(x) \subset V$, there exists $G \in \beta(X, x)$ such that $F(G) \subset V$. Set $U=G \cap U_{\alpha}$, then we have $U \in \beta\left(U_{\alpha}, x\right)$ (see [1], Lemma 2.5) and $\left(F / U_{\alpha}\right)(U)=F(U) \subset V$. Therefore, $F / U_{\alpha}$ is u. $\beta . c$. .

Sufficiency. Let $x \in X$ and $V$ be any open set of $Y$ such that $F(x) \subset V$. There exists $\alpha \in \nabla$ such that $x \in U_{\alpha}$. Since $F / U_{\alpha}$ is u. $\beta$.c. and $\left(F / U_{\alpha}\right)(x)=$ $F(x) \subset V$, there exists $U \in \beta\left(U_{\alpha}, x\right)$ such that $\left(F / U_{\alpha}\right)(U) \subset V$. Thus, we have $U \in \beta(X, x)$ (see [1], Lemma 2.7) and $F(U)=\left(F / U_{a}\right)(U) \subset V$. This shows that $F$ is u. $\beta . c$. . 
Theorem 3.10 Let $\left\{U_{\alpha}: \alpha \in \nabla\right\}$ be an $\alpha$-open cover of a topological space $X$. A multifunction $F: X \mapsto Y$ is l. $\beta$.c. if and only if the restriction $F / U_{\alpha}$ : $U_{\alpha} \mapsto Y$ is l.ß.c. for each $\alpha \in \nabla$.

Proof. The proof is similar to that of Theorem 3.9.

\section{Some properties}

Definition 4.1 A multifunction $F: X \mapsto Y$ is said to be upper rarely continuous [25] at a point $x$ of $X$ if for each open set $G$ of $Y$ containing $F(x)$, there exists a rare set $R_{G}$ with $C l\left(R_{G}\right) \cap G=\emptyset$ and an open set $U$ containing $x$ such that $F(U) \subset G \cup R_{G}$. A multifunction is said to be upper rarely continuous if it has the property at each point of $X$.

Theorem 4.1 If a multifunction $F: X \mapsto Y$ is upper rarely continuous at each point $x \in X$ and for each open set $G$ containing $F(x), F^{-}\left(C l\left(R_{G}\right)\right)$ is a $\beta$-closed set of $X$, where $R_{G}$ is the rare set of Definition 4.1, then $F$ is u. $\beta . c$.

Proof. Let $x \in X$ and $G$ be an open set such that $F(x) \subset G$. Since $F$ is upper rarely continuous, there exist an open set $V$ of $X$ containing $x$ and a rare set $R_{G}$ with $C l\left(R_{G}\right) \cap G=\emptyset$ such that $F(V) \subset G \cup R_{G}$. Let $U=$ $V \cap\left(X \backslash F^{-}\left(\mathrm{Cl}\left(R_{G}\right)\right)\right)$. Then we have $U \in \beta(X)$ (see [3], Theorem 2.7) and $x \in U$, since $x \in V$ and $x \in X \backslash F^{-}\left(\mathrm{Cl}\left(R_{G}\right)\right)$. If we suppose that $x \in F^{-}\left(\mathrm{Cl}\left(R_{G}\right)\right)$ then $F(x) \cap \mathrm{Cl}\left(R_{G}\right) \neq \emptyset$, but $F(x) \subset G$ and $G \cap \mathrm{Cl}\left(R_{G}\right)=\emptyset$. Let $s \in U$. Then $F(s) \subset G \cup R_{G}$ and $F(s) \cap \mathrm{Cl}\left(R_{G}\right)=\emptyset$. Therefore, we have $F(s) \cap R_{G}=\emptyset$, and hence $F(s) \subset G$. Since $U$ is a $\beta$-open set containing $x$, it

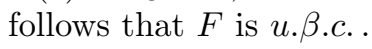

Definition 4.2 A multifunction $F: X \mapsto Y$ is said to be upper $\alpha$-continuous [18] if for each $x \in X$ and each open set $V$ of $Y$ containing $F(x)$, there exists an $\alpha$-open set $U$ containing $x$ such that $F(U) \subset V$.

Theorem 4.2 If $F, G: X \mapsto Y$ are multifunctions and $Y$ is a normal space such that

a) $F$ and $G$ are punctually closed;

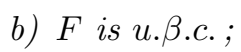

c) $G$ is upper $\alpha$-continuous,

then the set $\{x \in X: F(x) \cap G(x) \neq \emptyset\}$ is $\beta$-closed in $X$. 
Proof. Put $A=\{x \in X: F(x) \cap G(x) \neq \emptyset\}$ and let $x \in X \backslash A$. Then $F(x) \cap G(x)=\emptyset$. Since $Y$ is normal, there exist disjoint open sets $V$ and $W$ such that $F(x) \subset V$ and $G(x) \subset W$. Since $F$ is u. $\beta . c$., there exists $U_{1} \in$ $\beta(X, x)$ such that $F\left(U_{1}\right) \subset V$. Since $G$ is upper $\alpha$-continuous, there exists an $\alpha$-open set $U_{2}$ containing $x$ such that $G\left(U_{2}\right) \subset W$. Put $U=U_{1} \cap U_{2}$. Then $U \in \beta(X, x)$ (see [3], Corollary 2.14) and $F(U) \cap G(U)=\emptyset$. Therefore, we have $U \cap A=\emptyset$ and hence $A$ is $\beta$-closed in $X$.

Definition 4.3 The $\beta$-frontier of a subset $A$ of $X$, denoted by $\beta \operatorname{Fr}(A)$, is defined by $\beta \operatorname{Fr}(A)=\beta C l(A) \cap \beta C l(X \backslash A)=\beta C l(A)-\beta \operatorname{Int}(A)$.

Theorem 4.3 The set of all points $x$ of $X$ at which a multifunction $F: X \mapsto$

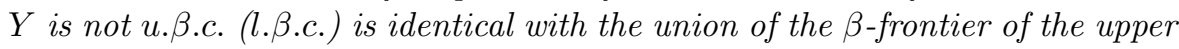
(lower) inverse images of open sets containing (meeting) $F(x)$.

Proof. Let $x$ be a point of $X$ at which $F$ is not u. $\beta . c$. . Then there exists an open set $V$ of $Y$ containing $F(x)$ such that $U \cap\left(X \backslash F^{+}(V)\right) \neq \emptyset$ for every $U \in \beta(X, x)$. Therefore, we have $x \in \beta \operatorname{Cl}\left(X \backslash F^{+}(V)\right)=X \backslash \beta \operatorname{Int}\left(F^{+}(V)\right)$ and $x \in F^{+}(V)$. Thus we obtain $x \in \beta \operatorname{Fr}\left(F^{+}(V)\right)$. Conversely, suppose that $V$ is an open set containing $F(x)$ and that $x \in \beta \operatorname{Fr}\left(F^{+}(V)\right)$. If $F$ is u. $\beta . c$, at $x$, then there exists $U \in \beta(X, x)$ such that $U \subset F^{+}(V)$; hence $x \in \beta \operatorname{Int}\left(F^{+}(V)\right)$. This

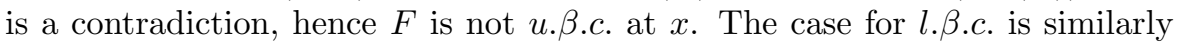
shown.

\section{$5 \beta$-continuity and quasi-continuity}

Definition 5.1 A multifunction $F: X \mapsto Y$ is said to be

(a) upper quasi continuous [23] if for each $x \in X$, each open set $U$ containing $x$ and each open set $V$ containing $F(x)$, there exists a nonempty open set $G$ of $X$ such that $G \subset U$ and $F(G) \subset V$;

(b) lower quasi continuous [23] if for each $x \in X$, each open set $U$ containing $x$ and each open set $V$ such that $F(x) \cap V \neq \emptyset$, there exists a nonempty open set $G$ of $X$ such that $G \subset U$ and $F(g) \cap V \neq \emptyset$ for every $g \in G$.

Lemma 5.1 (Noiri and Popa [21]) If $A$ is an $\alpha$-regular set of a topological space $X$, then for every open set $U$ which intersects $A$ there exists an open set $U_{A}$ such that $A \cap U_{A} \neq \emptyset$ and $C l\left(U_{A}\right) \subset U$.

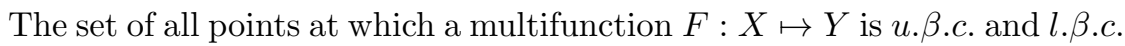
(resp. upper quasi continuous and lower quasi continuous) will be denoted by $B_{F}\left(\right.$ resp. $\left.Q_{F}\right)$. 
Theorem 5.1 If a multifunction $F: X \mapsto Y$ is punctually $\alpha$-regular and $\alpha$-paracompact, then $B_{F} \cap \operatorname{Int}\left(C l\left(Q_{F}\right)\right) \subset Q_{F}$.

Proof. Let $x \in B_{F} \cap \operatorname{Int}\left(\mathrm{Cl}\left(Q_{F}\right)\right)$. First, we show that $F$ is upper quasi continuous. Let $U$ and $V$ be open sets such that $x \in U$ and $F(x) \subset V$. Since $F(x)$ is $\alpha$-regular and $\alpha$-paracompact, by Lemma 3.2 there exists an open set $W$ such that $F(x) \subset W \subset \mathrm{Cl}(W) \subset V$. The upper $\beta$-continuity of $F$ at $x$ implies that there exists a nonempty open set $G \subset U \cap \operatorname{Int}\left(\mathrm{Cl}\left(Q_{F}\right)\right)$ such that $G \subset \mathrm{Cl}\left(F^{+}(W)\right)$. It follows from $G \subset \operatorname{Int}\left(\operatorname{Cl}\left(Q_{F}\right)\right)$ that $G \cap Q_{F} \neq \emptyset$.

$$
\text { If } s \in G \cap Q_{F} \text {, then } s \in F^{+}(\mathrm{Cl}(W)) \text {. }
$$

Suppose that (1) does not hold. Then there exists $s \in G \cap Q_{F}$ such that $s \in F^{-}(Y \backslash \mathrm{Cl}(W))$. The lower quasi continuity of $F$ implies that there exists a nonempty open set $G_{1} \subset G$ such that $G_{1} \subset F^{-}(Y \backslash \mathrm{Cl}(W)) \subset F^{-}(Y \backslash W)$ (see [24], Theorem 2.2). This contradicts that $G \subset \mathrm{Cl}\left(F^{+}(W)\right.$ ). It follows from (1) that if $s \in G \cap Q_{F}$ then $F(s) \subset \mathrm{Cl}(W) \subset V$. The upper quasi continuity of $F$ at $s$ implies that there exists a nonempty open set $H \subset U$ such that $F(H) \subset V$. Thus $F$ is upper quasi continuous at $x$. Next, we show that $F$ is lower quasi continuous. Let $U$ and $V$ be open sets such that $x \in U$ and $F(x) \cap V \neq \emptyset$. Since $F(x)$ is $\alpha$-regular, by Lemma 5.1 there exists an open set $W$ such that $F(x) \cap W \neq \emptyset$ and $\mathrm{Cl}(W) \subset V$. The lower $\beta$-continuity of $F$ at $x$ implies that there exists a nonempty open set $G \subset U \cap \operatorname{Int}\left(\mathrm{Cl}\left(Q_{F}\right)\right)$ such that $G \subset \mathrm{Cl}\left(F^{-}(W)\right)$. It follows from $G \subset \operatorname{Int}\left(\operatorname{Cl}\left(Q_{F}\right)\right)$ that $G \cap Q_{F} \neq \emptyset$.

$$
\text { If } s \in G \cap Q_{F} \text { then } s \in F^{-}(\mathrm{Cl}(W)) \text {. }
$$

Suppose that (2) does not hold. Then, there exists $s \in G \cap Q_{F}$ such that $s \in$ $F^{+}(Y \backslash \mathrm{Cl}(W))$. The upper quasi continuity of $F$ at $s$ implies that there exists a nonempty open set $G_{2} \subset G$ such that $G_{2} \subset F^{+}(Y \backslash \mathrm{Cl}(W)) \subset F^{+}(Y \backslash W)$ (see [24], Theorem 2.1). This is in contradiction with $G \subset \mathrm{Cl}\left(F^{-}(W)\right)$. It follows from (2) that if $s \in G \cap Q_{F}$, then $F(s) \cap \mathrm{Cl}(W) \neq \emptyset$ and hence $F(s) \cap V \neq \emptyset$. The lower quasi continuity of $F$ are $s$ implies that there exists a nonempty open set $H \subset U$ such that $F(H) \cap V \neq \emptyset$ for each $h \in H$. Thus $F$ is lower quasi continuous at $x$. Consequently we obtain $x \in Q_{F}$.

Corollary 5.1 (Borsik and Doboš [7]) Let $Y$ be a regular space and $f$ : $X \mapsto Y$ be a function. Then $B_{f} \cap \operatorname{Int}\left(C l\left(Q_{f}\right) \subset Q_{f}\right.$.

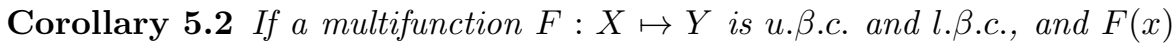
is $\alpha$-regular $\alpha$-paracompact for each $x \in X$, then $Q_{F}$ is semi-closed in $X$.

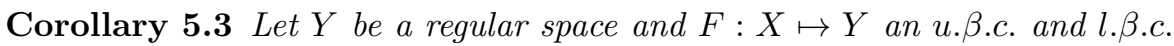
multifunction. If $F(x)$ is compact for each $x \in X$, then $Q_{F}$ is semi-closed in $X$. 
Corollary 5.4 (Borsik and Doboš [7]) If $Y$ is a regular space and $f$ : $X \mapsto Y$ a $\beta$-continuous function, then $Q_{f}$ is semi-closed in $X$.

Theorem 5.2 Let $F: X \mapsto Y$ be a multifunction such that $F(x)$ is $\alpha$-regular $\alpha$-paracompact for each $x \in X$. Then $F$ is upper and lower quasi continuous

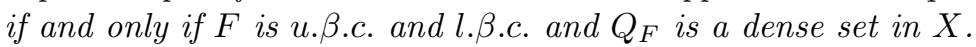

Corollary 5.5 (Borsiḱ and Doboš [7]) Let $Y$ be a regular space. Then $f$ : $X \mapsto Y$ is quasi continuous if and only if it is $\beta$-continuous and $Q_{f}$ is dense in $X$.

Definition 5.2 A multifunction $F: X \mapsto Y$ is said to be upper (resp. lower) s-quasi continuous [13] at a point $x \in X$ if for each open set $V$ of $Y$ containing (resp. meeting) $F(x)$ and having the connected complement, there exists $U \in$ $S O(X, x)$ such that $F(U) \subset V$ (resp. $\left.U \subset F^{-}(V)\right)$.

By $Q_{s}(F)$ we shall denote the set of all points of $X$ at which a multifunction $F: X \mapsto Y$ is upper and lower $s$-quasi continuous.

Theorem 5.3 Let $Y$ be a locally connected regular space. If $F: X \mapsto Y$ is a multifunction such that $F(x)$ is connected and compact for each $x \in X$, then $B_{F} \cap \operatorname{Int}\left(C l\left(Q_{s}(F)\right)\right) \subset Q_{s}(F)$.

Proof. The proof is similar to that of Theorem 5.1 and is thus omitted.

Corollary 5.6 Let $Y$ be a locally connected regular space. If a multifunction $F: X \mapsto Y$ is u. $\beta$.c. and l. $\beta$.c. and $F(x)$ is connected compact for each $x \in X$, then $Q_{s}(F)$ is semi-closed in $X$.

\section{Nets for multifunctions}

In what follows $(D,>)$ is a directed set, $\left(F_{\alpha}\right)$ is a net of multifunctions $F_{\alpha}$ : $X \mapsto Y, \alpha \in D$ and $F$ is a multifunction on $X$ into $Y$.

Definition 6.1 (1) $\left(F_{\alpha}\right)$ converges upper pointwise to $F$ on $X[8]$ if for each $x \in X$ and each open set $G \subset Y$ containing $F(x)$, there exists $\beta(x, G) \in D$ such that $F_{\alpha}(x) \subset G$ for all $\alpha>\beta(x, G)$;

(2) $\left(F_{\alpha}\right)$ converges lower pointwise to $F$ on $X$ [8] if for each $x \in X$ and each open set $G \subset Y$ which intersects $F(x)$, there exists $\beta(x, G) \in D$ such that $F_{\alpha}(x) \cap G \neq \emptyset$ for all $\alpha>\beta(x, G)$;

(3) $\left(F_{\alpha}\right)$ converges pointwise to $F$ on $X[8]$ if it converges upper pointwise and lower pointwise to $F$. 
Definition 6.2 (1) $\left(F_{\alpha}\right)$ converges quasi upper r-uniformly (q.u.r.u.) to $F$ on $X[8]$ if

(i) $\left(F_{\alpha}\right)$ converges pointwise to $F$ on $X$,

(ii) for each open set $G$ of $Y$ with $F^{+}(G) \neq \emptyset$ and each $\beta \in D$, there exists $\alpha>\beta$ such that $F_{\alpha}(x) \subset G$ for all $x \in F^{+}(G)$;

(2) $\left(F_{\alpha}\right)$ converges quasi lower r-uniformly (q.l.r.u.) to $F$ on $X[8]$ if

(i) $\left(F_{\alpha}\right)$ converges pointwise to $F$ on $X$,

(ii) for each open set $G$ of $Y$ with $F^{-}(G) \neq \emptyset$ and each $\beta \in D$, there exists $\alpha>\beta$ such that $F_{\alpha}(x) \cap G \neq \emptyset$ for all $x \in F^{-}(G)$;

(3) $\left(F_{\alpha}\right)$ converges quasi r-uniformly (q.r.u.) to $F$ on $X$ if it converges q.u.r.u. and q.l.r.u. .

Theorem 6.1 Let $\left(F_{\alpha}\right)$ be a net which converges q.l.r.u. to $F: X \mapsto Y$ and $F(x)$ be compact for each $x \in X$. If $Y$ is regular and $F_{\alpha}$ is u. $\beta$.c. for each $\alpha \in D$, then $F$ is u. $\beta . c$. .

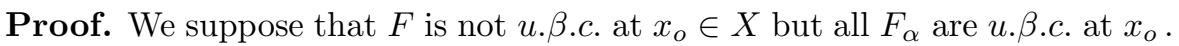
Then there exists an open set $G$ of $Y$ containing $F\left(x_{o}\right)$ such that for every $\beta$-open set $V$ of $X$ containing $x_{o}$, there exists $x_{V} \in V$ such that $F\left(x_{V}\right)$ is not contained in $G$. But $F\left(x_{o}\right) \cap(Y \backslash G)=\emptyset, F\left(x_{o}\right)$ is compact, $Y \backslash G$ is closed and $Y$ is regular. Therefore, it follows that there exist two disjoint open sets $G_{1}$ and $G_{2}$ such that $F\left(x_{o}\right) \subset G_{1}, Y \backslash G \subset G_{2}$. From the pointwise convergence of $\left(F_{\alpha}\right)$ to $F$ it follows that there exists $\alpha_{o} \in D$ such that $F_{\alpha}\left(x_{o}\right) \subset G_{1}$ for all $\alpha>\alpha_{o}$. But $F^{-}\left(G_{2}\right) \neq \emptyset$ since $x_{V} \in F^{-}\left(G_{2}\right)$ and $\left(F_{\alpha}\right)$ converges q.l.r.u. to $F$. Therefore, it follows that there exists $\gamma>\alpha_{o}$ such that $F_{\gamma}(x) \cap G_{2} \neq \emptyset$ for each $x \in F^{-}\left(G_{2}\right)$; hence $F_{\gamma}\left(x_{V}\right) \cap G_{2} \neq \emptyset$. This implies that $F_{\gamma}\left(x_{V}\right)$ is not contained in $G_{1}$. Therefore, $F_{\gamma}$ is not u. $\beta . c$. in $x_{o}$. This contradicts the hypothesis.

Theorem 6.2 Let $\left(F_{\alpha}\right)$ be a net which converges q.u.r.u. to $F: X \mapsto Y$. If

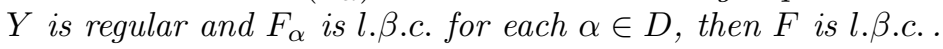

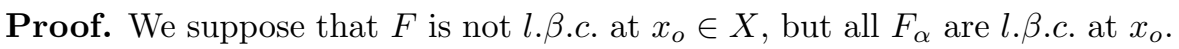
Then there exists an open set $G$ of $Y$ intersecting $F\left(x_{o}\right)$ such that for every $\beta$ open set $V$ of $X$ containing $x_{o}$, there exists $x_{V} \in V$ such that $F\left(x_{V}\right) \cap G=\emptyset$. Let $y_{o}$ be an arbitrary point of $F\left(x_{o}\right) \cap G$. Then $y_{o} \in Y \backslash(Y \backslash G)$ and $Y$ is regular. Therefore, it follows that there exist two disjoint open sets $G_{1}$ and $G_{2}$ such that $y_{o} \in G_{1}, Y \backslash G \subset G_{2}$. Hence $F\left(x_{o}\right) \cap G_{1} \neq \emptyset$. ¿From the pointwise convergence of $\left(F_{\alpha}\right)$ to $F$ it follows that there exists $\alpha_{o} \in D$ such 
that $F_{\alpha}\left(x_{o}\right) \cap G_{1} \neq \emptyset$ for all $\alpha>\alpha_{o}$. But $F^{+}\left(G_{2}\right) \neq \emptyset$ since $x_{V} \in F^{+}\left(G_{2}\right)$ and $\left(F_{\alpha}\right)$ converges q.u.r.u. to $F$. Therefore, it follows that there exists $\gamma>\alpha_{o}$ such that $F_{\gamma}(x) \subset G_{2}$ for each $x \in F^{+}\left(G_{2}\right)$; hence $F_{\gamma}\left(x_{V}\right) \subset G_{2}$. This implies that $F_{\gamma}\left(x_{v}\right) \cap G_{1}=\emptyset$. Therefore, $F_{\gamma}$ is not l.ß.c. in $x_{o}$. This contradicts the hypothesis.

Definition 6.3 Let $\left(F_{\alpha}\right)_{\alpha \in D}$ be a net of multifunctions on $X$ into $Y$. A multifunction $F^{*}: X \mapsto Y$ defined as follows: for each $x \in X, F^{*}(x)=\{y \in$ $Y:$ for each open neighborhood of $y$ and each $\beta \in D$, there exists $\alpha \in D$ such that $\alpha>\beta$ and $\left.V \cap F_{\alpha}(x) \neq \emptyset\right\}$ is called the upper topological limit [5] of the net $\left(F_{\alpha}\right)$.

Definition 6.4 A net $\left(F_{\alpha}\right)_{\alpha \in D}$ is said to be equally u.ß.c. at $x_{o} \in X$ if for every open set $V_{\alpha}$ containing $F_{\alpha}\left(x_{o}\right)$ there exists a $\beta$-open set $U$ containing $x_{o}$ such that $F_{\alpha}(U) \subset V_{\alpha}$ for all $\alpha \in D$.

Theorem 6.3 Let $\left(F_{\alpha}\right)_{\alpha \in D}$ be a net of multifunctions from a topological space $(X, \tau)$ into a compact topological space $(Y, \sigma)$. If the following are satisfied:

(1) $\cap\left\{\left(Y \backslash F_{\beta}(x)\right): \beta>\alpha\right\} \in \sigma$ for each $\alpha \in D$ and each $x \in X$,

(2) $\left(F_{\alpha}\right)$ is equally u.ß.c. on $X$,

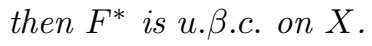

Proof. It is known that $F^{*}(x)=\cap\left\{\mathrm{Cl}\left(\cup\left\{F_{\beta}(x): \beta>\alpha\right\}\right): \alpha \in D\right\}$. From (1) we have $F^{\star}(x)=\cap\left\{\left[\cup\left\{F_{\beta}(x): \beta>\alpha\right\}\right]: \alpha \in D\right\}$. Since the net $\left(\cup\left\{F_{\beta}(x): \beta>\alpha\right\}\right)_{\alpha \in D}$ is a family of closed sets having the finite intersection property and $Y$ is compact, it follows that $F^{*}(x) \neq \emptyset$ for each $x \in X$. Now, let $x_{o} \in X$ and let $V \in \sigma$ such that $V \neq Y$ and $F^{*}\left(x_{o}\right) \subset V$. Then $F^{*}\left(x_{o}\right) \cap(Y \backslash$ $V)=\emptyset, F^{*}\left(x_{o}\right) \neq \emptyset$ and $Y \backslash V \neq \emptyset$. It results that $\cap\left\{\left[\cup\left\{F_{\beta}\left(x_{o}\right): \beta>\alpha\right\}\right]\right.$ : $\alpha \in D\} \cap(Y \backslash V)=\emptyset$ and hence $\cap\left\{\left[\cup\left\{F_{\beta}\left(x_{o}\right) \cap(Y \backslash V): \beta>\alpha\right\}\right]: \alpha \in D\right\}=\emptyset$. Since $Y$ is compact and the family $\left\{\left[\cup\left\{F_{\beta}\left(x_{o}\right) \cap(Y \backslash V): \beta>\alpha\right\}\right]: \alpha \in D\right\}$ is a family of closed sets with the empty intersection, there exists $\alpha \in D$ such that for each $\beta \in D$ with $\beta>\alpha$ we have $F_{\beta}\left(x_{o}\right) \cap(Y \backslash V)=\emptyset$; hence $F_{\beta}\left(x_{o}\right) \subset V$. Since the net $\left(F_{\alpha}\right)_{\alpha \in D}$ is equally u. $\beta$.c. on $X$, it results that there exists a $\beta$-open set $U$ containing $x_{o}$ such that $F_{\beta}(U) \subset V$ for each $\beta>\alpha$; hence $F_{\beta}(x) \cap(Y \backslash V)=\emptyset$ for each $x \in U$. Then we have $\cup\left\{F_{\beta}(x) \cap(Y \backslash V)\right.$ : $\beta>\alpha\}=\emptyset$; hence $\cap\left\{\left[\cup\left\{F_{\beta}(x): \beta>\alpha\right\}\right]: \alpha \in D\right\} \cap(Y \backslash V)=\emptyset$. This implies that $F^{*}(U) \subset V$. If $V=Y$ then it is clear that for each $\beta$ - open set $U$ containing $x_{o}$ we have $F^{*}(U) \subset V$. Hence $F^{*}$ is u. $\beta$.c. at $x_{o}$. Since $x_{o}$ is arbitrary, the proof is complete. 


\section{References}

[1] M. E. Abd El-Monsef, S. N. El-Deeb, and R. A. Mahmoud, $\beta$-open sets and $\beta$-continuous mappings, Bull. Fac. Sci. Assiut Univ. 12 (1983), 77-90.

[2] M. E. Abd El-Monsef, R. A. Mahmoud, and E. R. Lashin, $\beta$-closure and $\beta$-interior, J. Fac. Ed. Ain Shans Univ. 10 (1986), 235-245.

[3] D. Andrijević, Semi-preopen sets, Mat. Vesnik 38 (1986), 24-32.

[4] T. Banzaru, Multifunctions and M-product spaces, Bul. St. Tehn. Inst. Politeh. "T. Vuia" Timişoara Mat. Fiz. Mec. Teor. Apl. 17 (31) (1972), 17-23, in Romanian.

[5] T. Banzaru, On the upper semicontinuity of the upper topological limit for multifunction nets, Semin. Mat. Fiz. Inst. Politeh. "T. Vuia" Timişoara (1983), 59-64.

[6] J. Borsík, On almost quasicontinuous functions, Math. Bohemica 118 (1993), 241-248.

[7] J. Borsík and J. Dobos,, On decompositions of quasicontinuity, Real Anal. Exchange 16 (1990-1991), 292-305.

[8] N. Crivat and T. Banzaru, On the quasi continuity of the limit for nets of multifunctions, Semin. Mat. Fiz. Inst. Politeh. "T. Vuia" Timişoara (1983), 37-40.

[9] S. G. Crossley and S. K. Hildebrand, Semi-closure, Texas J. Sci 22 (1971), 99-112.

[10] N. El-Deeb, I. A. Hasanein, A. S. Mashhour, and T. Noiri, On p-regular spaces, Bull. Math. Soc. Sci. Math. R. S. Roumaine 27 (75) (1983), 311315 .

[11] J. Ewert, On almost quasicontinuity of functions, Tatra Mountains Math. Publ. 2 (1993), 81-92.

[12] J. Ewert, Almost quasicontinuity of multivalued maps, Math. Slovaka (1996), to appear.

[13] J. Ewert and T. Lipski, On S-quasi-continuous multivalued maps, Univ. u Novom Sadu, Zb. Rad. Prirod. Mat. Fak. Ser. Mat. 20 (1990), 167-183.

[14] I. Kovacević, Subsets and paracompactness, Univ. u Novom Sadu, Zb. Rad. Prirod.-Mat. Fac. Ser. Mat. 14 (1984), 79-87. 
[15] N. Levine, Semi-open sets and semi-continuity in topological spaces, Amer. Math. Monthly 70 (1963), 36-41.

[16] S. Marcus, Sur les fonctions quasicontinues au sens de S. Kempisty, Colloq. Math. 8 (1961), 47-53.

[17] A. S. Mashhour, M. E. Abd El-Monsef, and S. N. El-Deeb, On precontinuous and weak precontinuous mappings, Proc. Math. Phys. Soc. Egypt 53 (1982), 47-53.

[18] T. Neubrunn, Strongly quasi-continuous multivalued mappings, General Topology and its relation to Modern Analysis and Algebra (VI) (Prague 1986), Heldermann, Berlin, 1988, 351-359.

[19] O. Njåstad, On some classes of nearly open sets, Pacific J. Math. 15 (1965), 961-970.

[20] T. Noiri, On $\alpha$-continuous functions, Časopis Pěst. Math. 109 (1984), $118-126$.

[21] T. Noiri and V. Popa, On upper and lower weakly quasi-continuous multifunctions, Rev. Roumaine Pure Appl. 37 (1992), 449-508.

[22] T. Noiri and V. Popa, Almost weakly continuous multifunctions, Demonstratio Math. 26 (1993), 363-380.

[23] V. Popa, On a decomposition of quasicontinuity for multifunctions, Stud. Cerc. Mat. 27 (1975), 323-328, in Romanian.

[24] V. Popa, Sur certaines formes faibles de continuite pour les multifonctions, Rev. Roumaine Math. Pure Appl. 30 (1985), 539-546.

[25] V. Popa, Some properties of rarely continuous multifunctions, Conf. Nat. Geom. Topologie, 1988, Univ. Al. I. Cuza Iaşi (1989), 269-274.

[26] V. Popa and T. Noiri, On $\beta$-continuous functions, Real Anal. Exchange 18 (1992-1993), 544-548.

[27] V. Popa and T. Noiri, On upper and lower $\alpha$-continuous multifunctions, Math Slovaka 43 (1993), 477-491.

[28] D. Wine, Locally paracompact spaces, Glasnik Mat. 10 (30) (1975), 351357. 